\section{Gesundheitswesen: Qualität und Ökonomie sind vereinbar}

\author{
J. H. Dunant
}

Während Jahrtausenden waren Probleme des Gesundheitswesens in erster Linie Sache der Patienten, später von Heilern, dann der Ärzteschaft und der Universitäten, seit Mitte des 20. Jahrhunderts zunehmend auch von Versicherern. Heute haben Ökonomen, Sozialwissenschaftler, Versicherer, geschäftliche Interessengruppen und nicht zuletzt der Staat ein gewichtiges Wort mitzureden. Die meisten politischen Entscheidungen betreffen heute vor allem ökonomische Faktoren und weniger Qualitätskriterien. Ein typisches Beispiel war der Versuch der ClintonAdministration, eine "Gesundheitsreform" einzuleiten. Hier wurde mit einem rigiden Plan versucht, das Gesundheitswesen auf rein ökonomische Weise zu reorganisieren. Ärzte und Ärzteorganisationen waren von Anfang an keine Verhandlungspartner. Der Versuch scheiterte kläglich.

In der Schweiz wird 10\% des Bruttoinlandproduktes für das Gesundheitswesen ausgegeben, ein sehr grosser Betrag. Die Ausgaben für Gesundheit in der Schweiz sind um 37\% höher als im Durchschnitt von 23 vergleichbaren fortschrittlichen Ländern. Die eigentlichen Kosten der Medizin sind allerdings wesentlich geringer. Wohl beeinflussen die Ärzte ungefähr 75\% der Gesundheitskosten, viele dieser Kosten sind jedoch durch Produkte und durch andere Dienste sowie vor allem auch durch Löhne aller im Gesundheitswesen Angestellten verursacht. Gleichwohl wird immer wieder behauptet, dass der Grossteil der Kosten im Gesundheitswesen auf Kosten der Ärzte geht. Das Ärzteeinkommen ist für weniger als $12 \%$ der Gesamtkosten verantwortlich. Der Schweizer gibt für Ferien gleich viel aus wie für seine Gesundheit und fast doppelt so viel für sein Auto!

Eine Reihe von Umständen begünstigt einen erhöhten medizinischen Konsum. So sind für Angehörige verschiedener Berufe auf Weisung von Versicherungen, anderen Organisationen oder vom Staat periodische medizinische Untersuchungen vorgeschrieben. Die sogenannte defensive Chirurgie und Medizin des letzten Jahrzehnts und der Zukunft führt letzten Endes nicht selten zu höheren Kosten, bedingt durch mehrfache Untersuchungen und Eingriffe, aber

Korrespondenz:

PD Dr. med. Jean Henri Dunant, Nationalrat

Luftmattstrasse 12

CH-4052 Basel auch wegen Komplikationen im Zusammenhang mit Behandlungen. Die Bevölkerung über 65 Jahre wird jährlich weiter zunehmen. Dies verursacht auch eine stetige Zunahme der medizinischen Leistungen sowie der Löhne und Einkommen in diesem Bereich und damit selbstverständlich auch der Versicherungsprämien. Berücksichtigt man nur die medizinisch bedingte Zunahme der Gesundheitskosten, so sind jährliche Prämienerhöhungen von etwa 3\% zu erwarten, also eine 30\%ige Erhöhung in 10 Jahren.

Schliesslich werden die steigenden Prämien der Ärztehaftpflichtversicherungen auf die Honorare überwälzt, ebenfalls die Kosten der obligatorischen Fortbildung. Ein hoher Anteil der Gesundheitskosten entsteht also durch administrative, regulatorische, defensive und juristische Sachzwänge. Ausserdem können die Kosten für klinische Forschung und Ausbildung oft gar nicht von den eigentlichen Kosten der Medizin abgegrenzt werden.

Es wird immer wieder behauptet, dass unserer Bevölkerung zu viele Spezialisten und zu wenig Allgemeinpraktiker zur Verfügung stehen würden. In den 90er Jahren haben namentlich Krankenkassen und Politiker Strategien entwickelt, welche auf der Annahme beruhten, dass viele Spezialisten zu viele teure Untersuchungen und Operationen vornehmen würden. Gesamthaft gibt es in der Schweiz zu viele Ärzte, über das "zu viel» lässt sich jedoch streiten. Bezüglich Spezialisten dürfte die Zahl um 10\% "zu viel» liegen. Interessanterweise sind ungefähr 70\% aller Spezialisten ausserhalb operativer Fächer, z.B. in der Psychiatrie zu finden.

Die fälschliche Annahme, dass Spezialisten vor allem operativ tätig seien und deshalb massgeblich an den hohen Gesundheitskosten beteiligt sind, bekräftigt die Versicherer und Politiker in ihrer Vorstellung, dass die Gesundheitskosten durch eine Reduktion der Spezialistenzahl eingeschränkt werden könnten, namentlich durch Verminderung deren Einkommen und Umverteilung auf die Allgemeinpraktiker. In Tat und Wahrheit lehnt unsere Bevölkerung eine Behinderung des Zugangs zum Spezialisten ab.

In den letzten 20 Jahren wurden die Patienten immer mehr von der individuellen Beteiligung an den Kosten des Gesundheitswesens entlastet. Es war leicht voraussehbar, dass mit den zwangsläufig kostspieligen Fortschritten in der Diagnostik und Therapie, teuren Antibiotika, Krebsbehandlungen, Transplantationschirurgie, zunehmend grossen chirurgischen Eingriffen und Intensivpflege auch bei Patienten in weit fortgeschrittenem Alter die Kassen gezwungen sein würden, ihre Ausgaben einzudämmen. Finanzielle Aspekte und die Kontrollen durch die Kassen traten in den letzten Jahren vermehrt in den Vordergrund gegenüber dem freien Zugang zur Medizin, ihrer Qualität und ihrer Erfolge. Eine Strategie zur Sicherung langzeitiger Qualität schien weniger wichtig als die angestrebten Kostensenkungen und Umverteilungen. Zudem führte eine ruinöse Ausbau- und Mengenausweitungspolitik dazu, dass zunehmend eine Diskrepanz zwischen Finanzierungsmöglichkeiten und Finanzierungsbedarf entstand. 
Die Konsequenz davon wird sein, dass nicht mehr alle Patienten die ihnen angemessene, dem neuesten Stand der Medizin entsprechende Betreuung bekommen können. Wohl muss einer Zweiklassenmedizin entgegengewirkt werden, doch wird sich eine solche nur schwer vermeiden lassen. Ungerechtigkeiten müssen durch klare Prioritätensetzungen auf ein für alle vertretbares Mindestmass reduziert werden.

Einen nennenswerte Reduktion der Kosten wird nur schwerlich zu erreichen sein, ohne dass der hohe Stand des schweizerischen Gesundheitswesens leidet. Dennoch gibt es eine Reihe von Massnahmen, die im Zusammenhang mit einer Stabilisierung der Gesundheitskosten diskutiert und durch die Politik realisiert werden könnte. Parlament und Regierung müssen ein Finanzierungssystem suchen, das trotz steigendem Durchschnittsalter der Bevölkerung und trotz technischem Fortschritt den Anstieg der Kosten bremsen könnte. Bundesrat, Parlament und Versicherer glauben mehr an Kontrollen und Reglemente, welche die Gesundheitspolitik zunehmend zu einem dirigistischen Wirrwarr machen, und, ähnlich wie in der Landwirtschaftspolitik, niemals die Kostenexplosion eindämmen können.

\section{Welche Lösungsmöglichkeiten bieten sich an?}

Hier wäre zuerst am zentralen Punkt, am Patienten, anzusetzen. Seine Eigenverantwortung wird durch das KVG unterdrückt, er wird praktisch entmündigt. Langfristig sind Probleme des Gesundheitswesens nur zu lösen, wenn der Patient als aktiver, rational handelnder und eigenverantwortlicher Entscheidungsträger wieder die Rolle erhält, die ihm zusteht. Es kann nicht der Sinn einer sozialen Krankenversicherung sein, gewissermassen als "Vollkaskoversicherung" alle möglichen Risiken, Mittel und Therapien abzudecken.

Sowohl in der Grund- als auch in der Zusatzversicherung sollte der Patient an den Kosten der ihm zustehenden Leistung beteiligt werden. Bei der Grundversicherung wäre dies realisierbar mit Selbstbehalten, die in Funktion des steuerbaren Einkommens zunehmen. Einkommensabhängige Prämien, wie von der SP gefordert, wären nichts anders als eine Reichtumssteuer, die zur Kostendämpfung sicher nichts beitragen würde.

Die Prämien der Zusatzversicherung sollten nicht mehr eine Quersubventionierung der Grundversicherungsleistungen einschliessen. Damit würde die Zusatzversicherung günstiger. Ausserdem müssten individuell gestaltbare Produkte angeboten werden, damit sich der Mittelstand den Hauptvorteil der Zusatzversicherung, die freie Arztwahl, noch leisten kann. Heute ist das zum Teil schon nicht mehr der Fall.

Der Pflichtleistungskatalog der Grundversicherung sollte reduziert werden. Dazu muss allerdings dieser Katalog zuerst neu definiert werden, da er bisher praktisch alle Leistungen umfasst. Es sind Leistungen $\mathrm{zu}$ streichen, welche der einzelne selber bezahlen kann, ohne in eine finanzielle Notlage zu geraten. Solche Leistungen könnten ausserhalb der Grundversicherung versichert werden. Zahnarzt, Brille, Fitnessclub und alle nicht wissenschaftlich belegten Behandlungen sind selber zu bezahlen oder gehören ins Ressort von Zusatzversicherungen. Der Anreiz, medizinische Leistungen kostenbewusst zu konsumieren, muss gefördert werden. Höhere Wahlfranchisen sind vorzusehen. Beträge, die für Auto, Ferien, Freizeitgestaltung und Hobby problemlos ausgegeben werden, dürften auch für die eigene Gesundheit tragbar sein (Bezüger von Ergänzungsleistungen ausgenommen).

Der Wechsel von Zusatzversicherungen sollte erleichtert und der Bonus vom Folgeversicherer übernommen werden können.

Mit der Einführung eines persönlichen Gesundheitspasses (Chipcard), auf dem Resultate von Diagnoseleistungen wie z.B. Röntgen, Tomographien oder Laboranalysen und andere Daten gespeichert wären, könnten überflüssige Leistungen verhindert und ein verbesserter Informationsfluss gefördert werden. Die Chipcard könnte auch als medizinische Akte dienen. Die Einführung eines solchen Gesundheitspasses stösst gegenwärtig noch auf Widerstand, da Datenschutzprobleme bestehen und zahlreiche Leistungserbringer finanzielle Einbussen befürchten.

Schliesslich sind alternative Finanzierungen der Krankenversicherung $\mathrm{zu}$ prüfen mit individuellen Konti zugunsten des Versicherten (analog BVG). Ausgesteuerte Härtefälle müssten allerdings über einen Fonds abgesichert sein.

Auf der Angebotsseite müssen Einschränkungen vor allem im Spitalbereich stattfinden. Rationalisierungsmassnahmen sind in Zusammenarbeit über die Kantonsgrenzen hinweg zu ergreifen: Reduktion des Überangebots im Spitalbereich, vor allem Abbau von Doppelspurigkeiten durch Fusionen, Moratorium für die Zulassung weiterer Leistungserbringer, Schaffung gleicher Rahmenbedingungen für öffentliche und private Spitäler. Da auf Subventionen nicht verzichtet werden kann, sollte man sich darauf beschränken, Spitalleistungen (Patienten) zu subventionieren, nicht Objekte (Spitäler). Tagespauschalen sind durch Fallpauschalen zu ersetzen. Alle Spitäler haben eigenwirtschaftlich zu arbeiten, eine Privatisierung ist $\mathrm{zu}$ diskutieren.

Die 26 verschiedenen, zum Teil allzu ehrgeizigen Gesundheitssysteme der Schweiz sollten vermehrt regional zusammenarbeiten und besonders teure, seltene Behandlungen unter sich aufteilen. Die Frage der Fusion von Universitätszentren respektive medizinischen Fakultäten muss sorgfältig, unter Wahrnehmung der möglichen Nachteile, diskutiert werden. Es müsste also auf politischer Ebene ein Konsens unter den Kantonen und Landesregionen gefunden werden. Ansätze zu solchen Lösungen sind bereits vorhanden, grösstenteils mit Erfolg, namentlich was die Qualitätssteigerung, aber auch die Kostensenkung anbetrifft. Schliesslich sollte entschieden werden, in welcher Weise im Lande die teuersten Behandlungen (Spitzenmedizin) aufgeteilt und zentralisiert werden. 
Die Schweiz ist weltweit für ihren hohen Standard im Gesundheitswesen bekannt. Die Erhaltung dieses Qualitätsstandards sollte gegenüber ökonomischen Gesichtspunkten überwiegen. Drastische Massnahmen nur an einzelnen Bausteinen des sehr komplexen Gesundheitswesens würden unweigerlich zu schweren Störungen desselben und $\mathrm{zu}$ unverantwortlichen Ungerechtigkeiten führen. Die Zulassung ungenügend ausgebildeter Ärzte (Euro-Docs) und die weitere ungehemmte Zunahme von Praxiseröffnungen, gefördert durch den Kontrahierungszwang, sind abzulehnen. Die Schwächung des Kostenbewusstseins der Prämienzahler durch Begünstigung ungehemmter Konsumation medizinischer Leistungen infolge Abschaffung der Kopfprämien und Teilfinanzierung durch die Mehrwertssteuer würde schliesslich $\mathrm{zu}$ einer weiteren Kostenexplosion führen. Hingegen könnte eine weitere Zunahme der Kosten und damit der Prämien im bisherigen Umfang mit den weiter oben erwähnten Massnahmen gebremst werden, ohne Abstriche bei der Qualität in Kauf zu nehmen.

\section{Santé publique: la qualité et l'économie sont compatibles}

\author{
J. H. Dunant
}

Pendant des millénaires, les problèmes de la santé publique étaient l'affaire des malades, puis des guérisseurs, ensuite des médecins et universités et, depuis le $20^{\mathrm{e}}$ siècle, de plus en plus des assureurs. Aujourd'hui, les économistes, les spécialistes en sciences sociales, les assureurs, les organisations professionnelles et aussi l'état jouent un rôle important dans le domaine de la santé publique. La plupart des décisions politiques se fondent aujourd'hui avant tout sur des considérations économiques et de moins en moins sur le souci de la qualité. La tentative de l'administration Clinton de lancer une réforme de la santé publique américaine était un exemple typique à ce propos. En voulant réorganiser la santé publique selon des critères purement économiques qui excluaient d'emblée la participation des médecins et organisations médicales, les autorités américaines ont lamentablement échoué.

En Suisse, 10\% du produit intérieur brut sont dépensés pour la santé publique. Un montant énorme. Les dépenses pour la santé publique en Suisse dépassent en moyenne de $37 \%$ celles de 23 autres pays

Correspondance:

PD Dr Jean Henri Dunant, Conseiller national

Luftmattstrasse 12

CH-4052 Bâle au niveau économique comparable. Mais le coût à proprement parler de la médecine est nettement plus bas. Certes, les médecins influencent environ 75\% des frais de santé, mais une bonne partie de ces derniers découlent de produits et d'autres services ainsi que des salaires du personnel médical au sens large du terme. Néanmoins, on continue de prétendre que la majorité des coûts de la santé publique est due aux médecins. Le revenu des médecins ne représente que $12 \%$ des dépenses pour la santé. En moyenne, le Suisse dépense autant pour ses vacances que pour sa santé et sa voiture personnelle lui coûte même deux fois plus!

La consommation médicale est encouragée par plusieurs facteurs. Les assurances, d'autres institutions ou encore 1'Etat exigent que les personnes pratiquant certaines professions se soumettent périodiquement à un examen médical. La médecine et la chirurgie dites défensives qui sont apparues cette dernière décennie entraînent également une hausse des coûts en raison de la multiplication des interventions et aussi à la suite des complications provoquées par ces traitements. La population de plus de 65 ans ne cessera d'augmenter. Il s'en suivra forcément une multiplication des prestations médicales et, partant, une augmentation des revenus dans le secteur médical et des primes d'assurances. La hausse des coûts de la santé due uniquement aux interventions médicales provoque à elle seule une progression annuelle des primes d'environ 3\%, soit 30\% en dix ans.

L'augmentation des primes d'assurance-responsabilité civile des médecins est également reportée sur les honoraires, de même que le coût de la formation continue obligatoire. Une part importante du coût de la santé est donc générée par des obligations administratives, régulatrices, défensives et juridiques. En outre, il est souvent difficile de tracer la limite entre le coût de la recherche et la formation clinique, d'une part, et le coût à proprement parler de la médecine, d'autre part. 
On entend souvent la critique selon laquelle il y a trop de médecins spécialistes en Suisse alors que nous manquons de généralistes. Dans les années nonante, des caisses-maladies et des milieux politiques ont développé des stratégies basées sur l'hypothèse que nombre de spécialistes procèdent à des investigations et opérations trop onéreuses. Dans l'ensemble, il y a certes trop de médecins en Suisse, mais ce "trop" est sujet à controverse. S'agissant des spécialistes, on peut admettre qu'il y en a effectivement environ 10\% "de trop». Détail intéressant: environ 70\% des spécialistes travaillent dans des domaines où il n'y a pas d'opérations, soit notamment en psychiatrie.

L'hypothèse erronée selon laquelle les spécialistes procèdent surtout à des opérations et génèrent de ce fait une partie importante des coûts de la santé a confirmé les assureurs et milieux politiques dans leur idée qu'il suffirait de réduire le nombre de spécialistes pour abaisser les coûts. Une réduction qui se ferait par une baisse du revenu des spécialistes au profit des généralistes. Dans la réalité, cependant, la population refuse toute restriction de l'accès aux spécialistes.

Durant les vingt ans écoulés, les patients ont été constamment déchargés de leur participation individuelle aux coûts de la santé. Face au progrès des techniques médicales onéreuses dans le diagnostic et la thérapie - antibiotiques extrêmement chers, traitements du cancer, chirurgie de transplantation, multiplication de lourdes interventions chirurgicales et soins intensifs - dont profite la population jusqu'à un âge avancé, il était évident que les caisses-maladies allaient devoir prendre des mesures pour limiter leurs dépenses. Des critères financiers et des contrôles sévères imposés par les caisses ont pris le pas sur le libre accès à la médecine et sur le souci de la qualité des prestations médicales. Au lieu de développer une stratégie visant à assurer une qualité durable de la médecine, on s'est concentré sur la réduction des coûts et sur la mise en place de mécanismes de redistribution, et une politique ruineuse d'extension quantitative des prestations médicales a encore approfondi le clivage entre les charges et les possibilités de financement.

La conséquence de cette évolution est évidente: les patients n'auront plus tous accès aux traitements médicaux les plus modernes et les plus efficaces. Il faut certes tenter d'éviter l'avènement d'une médecine à deux classes, mais cela sera très difficile. Les injustices doivent être réduites à un minimum supportable par la fixation de priorités claires.

Il sera difficile de réduire notablement le coût de la santé publique sans que le niveau élevé de la médecine suisse n'en soit affecté. Il existe néanmoins un certain nombre de mesures de stabilisation des coûts qui devraient être débattues et imposées par les autorités politiques. Le Parlement et le Gouvernement doivent chercher ensemble un système de financement qui permet de freiner la hausse des coûts malgré l'augmentation de l'âge moyen de la population et malgré le progrès des techniques médicales. Actuellement, le Conseil fédéral, le Parlement et les assurances donnent trop de place aux contrôles et aux règlements qui compliquent la santé publique (par analogie à la politique agricole) sans pour autant endiguer l'explosion des coûts.

\section{Quelles possibilités s'offrent à nous?}

Il faut commencer par intervenir au cœur du problème, c'est-à-dire auprès des patients. La LAMal actuelle les empêche d'assumer leurs responsabilités individuelles. Ils sont quasiment mis sous tutelle. A long terme, les problèmes de la santé publique ne peuvent être résolus que si le patient peut jouer un rôle actif, rationnel et responsable, donc prendre les décisions qui lui reviennent. Le rôle d'une assurancemaladie sociale n'est pas de couvrir tous les risques et toutes les thérapies comme une casco complète.

Le patient devrait être appelé à participer au coût du traitement auquel il a droit tant dans l'assurance de base que dans l'assurance complémentaire. Dans l'assurance de base, ce principe pourrait être concrétisé par des franchises augmentant en fonction du revenu imposable. Les primes calculées en fonction $\mathrm{du}$ revenu selon la proposition du Parti socialiste ne constituent en fait qu'un impôt sur la richesse qui ne contribuerait nullement à la réduction des coûts.

Les primes pour l'assurance complémentaire ne devraient plus servir à subventionner l'assurance de base. Ainsi, l'assurance complémentaire deviendrait moins chère. En outre, les caisses seraient contraintes de proposer des produits d'assurance adaptés aux besoins individuels afin que les classes moyennes puissent encore profiter du principal avantage de l'assurance complémentaire, à savoir le libre choix du médecin. Cela n'est plus toujours le cas aujourd'hui.

La liste des prestations médicales obligatoirement couvertes par l'assurance de base doit être réduite. Mais, pour cela, il faut commencer par redéfinir cette liste qui aujourd'hui inclut presque toutes les prestations possibles. Il faut supprimer les prestations qu'un particulier peut payer lui-même sans risquer de rencontrer de gros problèmes financiers. Ces prestations peuvent être assurées en dehors de l'assurance de base. Le dentiste, les lunettes, le club de fitness ainsi que toutes les prestations qui ne sont pas scientifiquement fondées doivent être payés par le bénéficiaire ou par l'assurance complémentaire. Il faut d'une manière générale aiguiser la conscience du prix des prestations médicales. Les franchises facultatives doivent être augmentées. Les mêmes sommes dépensées pour la voiture, les vacances, les loisirs et les hobbies devraient aussi être supportables quand il s'agit de sa propre santé (exception faite des bénéficiaires de prestations complémentaires qui ont besoin d'un soutien supplémentaire).

Le changement d'assurance complémentaire doit être facilité et l'assuré doit pouvoir emporter son bonus dans sa nouvelle assurance. L'introduction d'un passeport-santé personnel (carte à puce) sur lequel figurent les résultats de diagnostics précédents (rayons X, tomographies, analyses de laboratoire et autres données) permettrait d'éviter des actes médi- 
caux inutiles et améliorerait le flux de l'information. Cette carte à puce pourrait également servir de dossier médical. Si cette méthode suscite encore des résistances, c'est pour des raisons de protection des données personnelles et aussi parce que certains fournisseurs de prestations médicales craignent pour leurs affaires.

Enfin, il convient d'étudier des nouveaux modes de financement de l'assurance-maladie avec des comptes individuels des assurées (par analogie à la LPP). Les cas difficiles devraient cependant être couverts par un fonds spécial.

Du côté, de l'offre médicale, il faut procéder à des restrictions notamment dans le secteur hospitalier. Des mesures de rationalisation doivent être prises au-delà des frontières cantonales: réduction de l'offre hospitalière excédentaire, suppression des doubles emplois par le biais de fusions, moratoire pour l'admission de nouveaux fournisseurs de prestations médicales, mise en place de conditions de travail égales pour les hôpitaux publics et privés. Les subventions restant indispensables, l'Etat doit se limiter à subventionner des prestations hospitalières (les patients) et non pas subventionner des objets (les hôpitaux). Les forfaits journaliers doivent être remplacés par des forfaits par cas. Tous les hôpitaux doivent travailler de manière à couvrir leurs frais et il convient aussi d'examiner la possibilité d'une privatisation.

Les 26 systèmes de santé publique que connaît la Suisse et qui manifestent souvent des ambitions excessives doivent être forcés à collaborer davan- tage et à répartir entre eux les traitements rares et onéreux. La question de la fusion des centres universitaires ou des facultés de médecine doit être examinée en détail. Il convient donc de trouver un consensus politique entre cantons et régions du pays. Des premières tentatives ont été faites, généralement avec des effets positifs aussi bien sur la qualité des soins que sur les coûts. Enfin, il faut décider de la répartition et de la centralisation des institutions pratiquant les traitements les plus onéreux (médecine de pointe).

La Suisse est connue dans le monde entier pour le niveau élevé de sa santé publique. La sauvegarde de ce standard doit passer avant les considérations économiques. Des interventions aussi massives que ponctuelles dans ce système complexe provoqueraient des disfonctionnements graves et des injustices inacceptables. L'admission de médecins insuffisamment formés ("Euro-Docs») et l'ouverture incontrôlée de nouveaux cabinets médicaux (notamment à cause de l'obligation de contracter faite aux assurances) doivent être refusées. Il faut absolument éviter de déresponsabiliser l'assuré et ne pas l'encourager à consommer des prestations médicales en supprimant le système actuel de la prime individuelle ou en prélevant une part du produit de la TVA pour financer la santé publique.

Les mesures décrites ci-dessus permettent de freiner la hausse actuelle des coûts de la santé et, partant, des primes d'assurance sans pour autant affecter la qualité des prestations médicales. 